\title{
LOW DIMENSIONAL CHAOS AND ASYMPTOTIC TIME BEHAVIOR IN THE MECHANICS OF FLUIDS
}

\author{
G. Boffetta ${ }^{1}$, G. Lacorata ${ }^{2}$ and A. Vulpiani ${ }^{3}$ \\ ${ }^{1}$ Dipartimento di Fisica Generale, INFM and INFN, Università di Torino, \\ Via Pietro Giuria 1, 10125 Torino, Italy \\ ${ }^{2}$ CNR - Istituto di Scienze dell'Atmosfera e del Clima, Sezione di Lecce, \\ Str. Pr. Lecce-Monteroni, 73100 Lecce, Italy \\ 3 Dipartimento di Fisica, INFM (UdR and CSM) and INFN, Università di Roma "la Sapienza", \\ Piazzale Aldo Moro 5, 00185 Roma, Italy
}

\section{INTRODUCTION}

The contribution of H.J. Poincaré in Celestial Mechanics has an extremely rare, possibly even unique, character in Physics and Mathematics. While, usually, scientific books become obsolete in a few years (or several tens of years), the Méthodes Nouvelles de la Mécanique Céleste [35] more than one century later still remains a source of inspiration not only for beginners but also for confirmed scientists in many branches of the Theory of Dynamical Systems. Many of the methods, concepts and ideas introduced there by Poincaré are still useful tools even in fields apparently distant from Celestial Mechanics.

We discuss here Chaotic Advection in laminar incompressible flows and long-time diffusive behavior. The basic mechanism for the chaotic behavior generated by homoclinic intersections in quasi-integrable Hamiltonian systems, as well as the multi-scale expansion, turn out to be still the main ingredients of this issue. The multi-scale approach for partial differential equations, which will be explained below, can be seen as a spatio-temporal extension of the methods introduced by Lindstedt, and improved by Poincaré, for the treatment of the secular terms in Mechanics. 


\section{LAGRANGIAN CHAOS}

A problem of great interest concerns the study of the spatial and temporal structure of the so-called passive fields, indicating by this term quantities passively driven by the flow (i.e. without perturbing the dynamics of the fluid), such as the temperature under certain conditions [31]. The equation for the evolution of a passive scalar field $\theta(\mathbf{x}, t)$, advected by a given velocity field $\mathbf{v}(\mathbf{x}, t)$, is

$$
\partial_{t} \theta+\nabla \cdot(\mathbf{v} \theta)=D \nabla^{2} \theta
$$

where $D$ is the molecular diffusion coefficient.

The problem (1) can be studied through two different approaches. Either one deals at any time $t$, and any point $\mathbf{x}$ of the space domain covered by the fluid, with the field $\theta(\mathbf{x}, t)$ or one deals with the trajectory of each fluid particle. The two approaches are usually designed as "Eulerian" and "Lagrangian", although both of them are due to Euler [23]. The two points of view are in principle equivalent.

The motion of a fluid particle is determined by the differential equation

$$
\frac{d \mathbf{x}}{d t}=\mathbf{v}(\mathbf{x}, t)
$$

which also describes the motion of test particles, for example a powder embedded in the fluid, provided that the particles are neutral and small enough not to perturb the velocity field, although large enough not to perform a Brownian motion. Particles of this type are commonly used for flow visualization in fluid mechanics experiments, see [39]. Let us remark that the precise equation for the motion of a material particle in a fluid can be rather complicated [30].

It is now well established that even in regular (i.e. laminar) velocity field the motion of fluid particles may be very irregular (i.e. turbulent) $[19,2]$. In this case, initially nearby trajectories diverge exponentially and one speaks of Lagrangian chaos. In general, chaotic behaviors can arise in two-dimensional flow only for time dependent velocity fields in two 
dimensions, while it can be present even for stationary velocity fields in three dimensions $[1,15]$. Let us observe that for the dynamical system (2) the phase space coincides with the physical space.

In the following we will consider only incompressible flows. If $D=0$, it is easy to realize that (1) is equivalent to (2). In fact, we can write

$$
\theta(\mathbf{x}, t)=\theta_{o}\left(T^{-t} \mathbf{x}\right)
$$

where $\theta_{o}(\mathbf{x})=\theta(\mathbf{x}, t=0)$ and $T$ is the formal evolution operator of $(2)$,

$$
\mathbf{x}(t)=T^{t} \mathbf{x}(0)
$$

Taking into account the molecular diffusion $D,(1)$ is nothing but the Fokker-Planck equation associated to the Langevin equation [13]

$$
\frac{d \mathbf{x}}{d t}=\mathbf{v}(\mathbf{x}, t)+\eta(t)
$$

where $\eta$ is a three-dimensional Gaussian process with zero mean and variance

$$
\left\langle\eta_{i}(t) \eta_{j}\left(t^{\prime}\right)\right\rangle=2 D \delta_{i j} \delta\left(t-t^{\prime}\right)
$$

In the following we will consider only incompressible flow

$$
\nabla \cdot \mathbf{v}=0
$$

for which the dynamical system (2) is conservative, i.e. the volume is preserved under the time evolution. In two dimensions, the constraint (7) is automatically satisfied assuming

$$
v_{1}=\frac{\partial \psi}{\partial x_{2}}, \quad v_{2}=-\frac{\partial \psi}{\partial x_{1}}
$$

where $\psi(\mathbf{x}, t)$ is the stream function, which is assumed to be sufficiently smooth. Inserting (8) into (2) the evolution equations become

$$
\frac{d x_{1}}{d t}=\frac{\partial \psi}{\partial x_{2}}, \quad \frac{d x_{2}}{d t}=-\frac{\partial \psi}{\partial x_{1}} .
$$

Formally (9) is a Hamiltonian system with the Hamiltonian given by the stream function $\psi$, and the canonical variables are the spatial coordinates $x_{1}$ and $x_{2}:(q, p)=\left(x_{1}, x_{2}\right)$. 


\section{A. Examples of Lagrangian chaos}

As a first example we consider a $3 D$ stationary velocity field, the so-called ABC flow

$$
\mathbf{v}=(A \sin z+C \cos y, B \sin x+A \cos z, C \sin y+B \cos x)
$$

where $A, B$ and $C$ are non zero real parameters. Because of the incompressibility condition, the evolution $\mathbf{x}(0) \rightarrow \mathbf{x}(t)$ defines a conservative dynamics (i.e. phase space volumes are preserved).

Arnold $[4,5]$ argued that (10) is a good candidate for chaotic motion, namely the trajectories of the dynamical system (2) may exhibit sensitivity to initial conditions. Let us briefly repeat his elegant argument. For a steady state solution of the $3 D$ Euler equation one has:

$$
\begin{aligned}
\nabla \cdot \mathbf{v} & =0 \\
\mathbf{v} \times(\nabla \times \mathbf{v}) & =\nabla \alpha \\
\alpha & =\frac{P}{\rho}+\frac{\mathbf{v}^{2}}{2}
\end{aligned}
$$

where $P$ is the pressure and $\rho$ the density. As a consequence of the Bernoulli theorem [24], $\alpha(\mathbf{x})$ is constant along a streamline - that is a Lagrangian trajectory $\mathbf{x}(t)$. One can easily verify that chaotic motion can appear only if $\alpha(\mathbf{x})$ is constant (i.e. $\nabla \alpha(\mathbf{x})=0$ ) in a part of the space. Otherwise the trajectory would be confined on a $2 D$ surface $\alpha(\mathbf{x})=$ constant, where the motion must be regular as a consequence of general arguments [34]. In order to satisfy such a constraint, from (11) one has the Beltrami condition:

$$
\nabla \times \mathbf{v}=\gamma(\mathbf{x}) \mathbf{v}
$$

Taking the divergence of both sides in (12), one gets : $0=\mathbf{v} \cdot \nabla \gamma$ and, once again, if $\gamma$ is not constant, the trajectory is confined on a $2 D$ surface $\gamma(\mathbf{x})=$ constant and the motion is not chaotic. Thus chaotic motion can appear only if we have (12) with $\gamma(\mathbf{x})=$ constant in a part of space: in this case, nothing more, it seems, compels the trajectory to stay on 
a surface. Arnold [1] concludes : "it is probable that [such] flows have trajectories with complicated topology. Such complications occur in celestial mechanics". (As we see, the work of Poincaré on celestial mechanics acts as a guide here). It is easy to verify that the field $\mathbf{v}$ given by (10) satisfies (12) with $\gamma(\mathbf{x})=$ constant (this example is given by Arnold himself in [1]), and, indeed, numerical experiments by Hénon [19] provided evidence that Lagrangian motion under velocity (10) is chaotic for typical values of the parameters $A, B$, and $C$ (see an example in Figure 1).

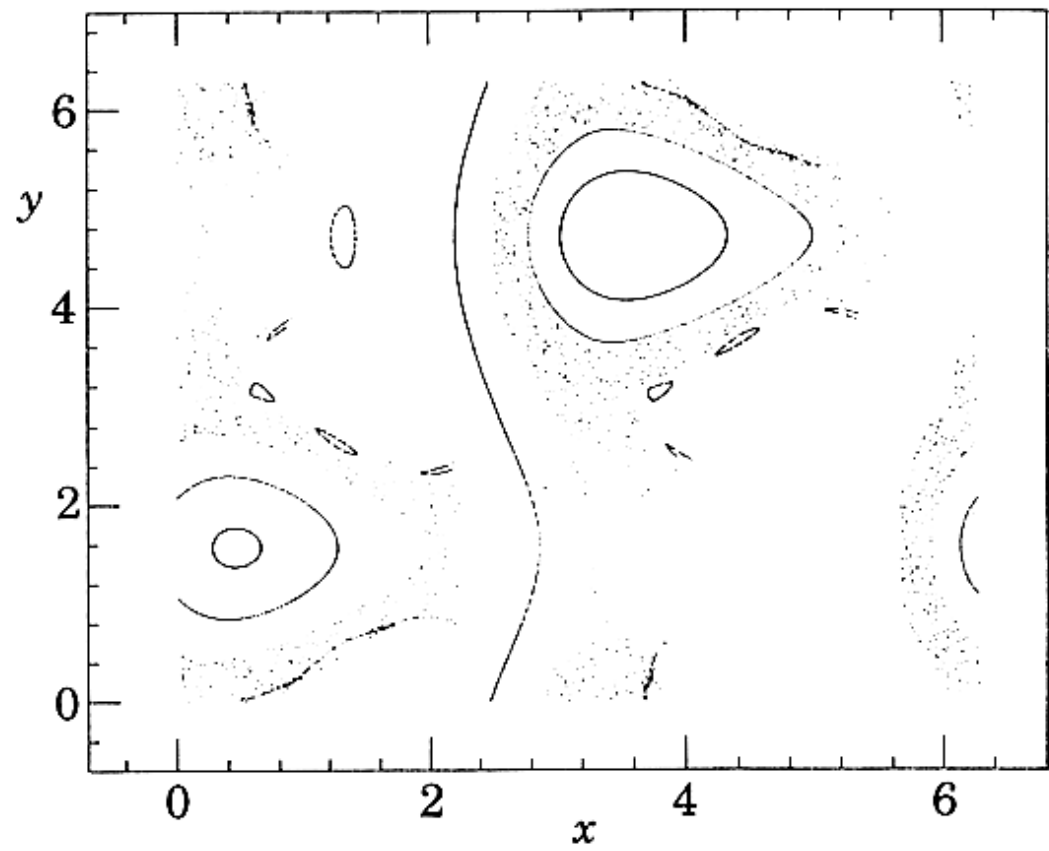

FIG. 1. Intersections with the Poincaré section, plane $z=0$, of eight trajectories of the $\mathrm{ABC}$ flow with parameters $A=2.0, B=1.70, C=1.50$.

Hénon wrote [19]: "In conclusion, one is led to this curious result that the particular case $[\alpha=$ const ., $\gamma=$ const. $]$ seems to have a topology much more complicated than the general case."

In a two-dimensional incompressible stationary flow the motion of fluid particles is given by a time independent one-dimensional Hamiltonian system (i.e. with two degrees of freedom) and, since trajectories follow iso- $\psi$ lines, it is impossible to have chaos. However, for 
explicit time dependent stream function $\psi$ the system (9) can exhibit chaotic motion [34]. Indeed, generically in one-dimensional Hamiltonian systems, a periodic perturbation gives origin to a so-called "stochastic layer" around the separatrices where the motion is chaotic, as consequence of unfolding and crossing of the stable and unstable manifolds in domains centered at the hyperbolic fixed points. This is the celebrated mechanism of the homoclinic intersection, formerly discovered by Poincaré in his work on Celestial Mechanics ${ }^{1}$.

In the particular case of time periodic velocity fields, $\mathbf{v}(\mathbf{x}, t+T)=\mathbf{v}(\mathbf{x}, t)$, the trajectory of (2) can be studied in terms of discrete dynamical systems: the position $\mathbf{x}(t+T)$ is determined in terms of $\mathbf{x}(t)$. The Poincaré map $\mathbf{x}(t) \rightarrow \mathbf{x}(t+T)$ will not depend explicitly on $t$ (given the position $\mathbf{x}(t)$ ) thus (2) can be written in the form

$$
\mathbf{x}(n+1)=\mathbf{F}[\mathbf{x}(n)]
$$

where now the time is measured in units of the period $T$. Because of incompressibility, the map (13) is conservative and the Lebesgue measure $d \mathbf{x}$ is invariant:

$$
|\operatorname{det} A[\mathbf{x}]|=1, \quad \text { where } \quad A_{i j}[\mathbf{x}]=\frac{\partial F_{i}[\mathbf{x}]}{\partial x_{j}} .
$$

The explicit form of $\mathbf{F}$ for a general $2 D$ or $3 D$ flow is usually very difficult to find. However, in some simple cases, this can be deduced on the basis of physical features $[3,12]$.

\section{EULERIAN PROPERTIES AND LAGRANGIAN CHAOS}

In principle, the evolution of the velocity field $\mathbf{v}$ is described by partial differential equations, e.g. Navier-Stokes or Boussinesq equations. However, often in weakly turbulent situations, a good approximation of the flow can be obtained by using a Galerkin approach i.e. writing the velocity field in terms of suitable functions (usually a Fourier series), and reducing the Eulerian problem which is, in principle, described by a partial differential equation, to a (small) system of $F$ ordinary differential equations [9,27]. It is possible to show

\footnotetext{
${ }^{1}$ See the chapter by François Beguin in this book
} 
that, under suitable conditions, this procedure (i.e. the approximation of an infinite dimensional system with a finite dimensional one) can be performed with full mathematical rigor [20]. The motion of a fluid particle is then described by the $(d+F)$-dimensional dynamical system

$$
\begin{array}{ll}
\frac{d \mathbf{Q}}{d t}=\mathbf{f}(\mathbf{Q}, t) & \text { with } \mathbf{Q}, \mathbf{f}(\mathbf{Q}, t) \in \mathbb{R}^{F} \\
\frac{d \mathbf{x}}{d t}=\mathbf{v}(\mathbf{x}, \mathbf{Q}) & \text { with } \mathbf{x}, \mathbf{v}(\mathbf{x}, \mathbf{Q}) \in \mathbb{R}^{d}
\end{array}
$$

where $d$ is the space dimensionality and $\mathbf{Q}=\left(Q_{1}, \ldots Q_{F}\right)$ are the $F$ variables (typically normal modes) which give a representation of the velocity field $\mathbf{v}$. Note that the equations (15) describe the Eulerian evolution and do not depend on the Lagrangian part (16), so (15) can be solved independently from (16).

In order to characterize the degree of chaos, three different Lyapunov exponents can be defined [17]:

- a) $\lambda_{\mathrm{E}}$ for the Eulerian part (15);

- b) $\lambda_{\mathrm{L}}$ for the Lagrangian part (16), where the time evolution of the velocity field (i.e. $\mathbf{Q}(\mathrm{t}))$ is assumed to be known;

- c) $\lambda_{\mathrm{T}}$ for the total system of the $d+F$ equations.

These Lyapunov exponents [15] are defined as:

$$
\lambda_{\mathrm{E}, \mathrm{L}, \mathrm{T}}=\lim _{t \rightarrow \infty} \frac{1}{t} \ln \frac{\left|\mathbf{z}(t)^{(\mathrm{E}, \mathrm{L}, \mathrm{T})}\right|}{\left|\mathbf{z}(0)^{(\mathrm{E}, \mathrm{L}, \mathrm{T})}\right|}
$$

where the limit in (17) exists almost everywhere. The evolution of the three tangent vectors $\mathbf{z}$ are given by the linearized stability equations for the Eulerian part, for the Lagrangian part and for the total system, respectively:

$$
\frac{d z_{i}^{(\mathrm{E})}}{d t}=\left.\sum_{j=1}^{F} \frac{\partial f_{i}}{\partial Q_{j}}\right|_{\mathbf{Q}(t)} z_{j}^{(\mathrm{E})}, \quad \mathbf{z}(t)^{(\mathrm{E})} \in \mathbb{R}^{F}
$$




$$
\begin{gathered}
\frac{d z_{i}^{(\mathrm{L})}}{d t}=\left.\sum_{j=1}^{d} \frac{\partial v_{i}}{\partial x_{j}}\right|_{\mathbf{x}(t)} z_{j}^{(\mathrm{L})}, \quad \mathbf{z}(t)^{(\mathrm{L})} \in \mathbb{R}^{d} \\
\frac{d z_{i}^{(\mathrm{T})}}{d t}=\left.\sum_{j=1}^{d+F} \frac{\partial G_{i}}{\partial y_{j}}\right|_{\mathbf{y}(t)} z_{j}^{(\mathrm{T})}, \quad \mathbf{z}(t)^{(\mathrm{T})} \in \mathbb{R}^{F+d}
\end{gathered}
$$

and $\mathbf{y}=\left(Q_{1}, \ldots, Q_{F}, x_{1}, \ldots, x_{d}\right)$ and $\mathbf{G}=\left(f_{1}, \ldots, f_{F}, v_{1}, \ldots, v_{d}\right)$. The meaning of these Lyapunov exponents is evident:

- a) $\lambda_{\mathrm{E}}$ is the mean exponential rate of the increasing of the uncertainty in the knowledge of the velocity field (which is represented by $\mathbf{Q}$ and is independent on the Lagrangian motion);

- b) $\lambda_{\mathrm{L}}$ estimates the rate at which the distance $\delta x(t)$ between two fluid particles initially close increases with time, when the velocity field is given, i.e. a particle pair in the same Eulerian realization (i.e. the same $\mathbf{Q}(t)$ );

- c) $\lambda_{\mathrm{T}}$ is the rate of growth of the distance between initially close particle pairs, when the velocity field is not known with infinite precision.

Thus two different dynamical systems can be defined: one for the evolution of the velocity field in the F-dimensional Fourier space; another one for the evolution of the Lagrangian trajectories in the $d$-dimensional physical space. Let us notice that, since the dynamical system (16) is conservative, one can have the coexistence of non communicating regions and the Lyapunov exponent can depend on the initial conditions (see below). We stress, at this regard, that there is no general relation between $\lambda_{\mathrm{E}}$ and $\lambda_{\mathrm{L}}$. One could expect that in presence of a chaotic velocity field the particle motion has to be chaotic. However, the inequality $\lambda_{\mathrm{L}} \geq \lambda_{\mathrm{E}}$ - even if generic - sometimes does not hold, e.g. in some systems like the Lorenz model [17] and in generic $2 D$ flows when the Lagrangian motion occurs around well defined vortex structures [6] as discussed in the following. On the contrary, one has [15]

$$
\lambda_{\mathrm{T}}=\max \left(\lambda_{\mathrm{E}}, \lambda_{\mathrm{L}}\right)
$$




\section{A. Lagrangian chaos in two-dimensional flows}

Let us now consider the two-dimensional Navier-Stokes equations with periodic boundary conditions at low Reynolds numbers, for which we can expand the stream function $\psi$ in Fourier series and take into account only the first $F$ terms $[9,27]$,

$$
\psi=-i \sum_{j=1}^{F} k_{j}^{-1} Q_{j} e^{i \mathbf{k}_{j} \mathbf{x}}+\text { c.c. },
$$

where c.c. indicates the complex conjugate term and $\mathbf{Q}=\left(Q_{1}, \ldots, Q_{F}\right)$ are the Fourier coefficients. Inserting (22) into the Navier-Stokes equations and by an appropriate time rescaling, we obtain the system of $F$ ordinary differential equations

$$
\frac{d Q_{j}}{d t}=-k_{j}^{2} Q_{j}+\sum_{l, m} A_{j l m} Q_{l} Q_{m}+f_{j},
$$

in which $f_{j}$ represents an external forcing. Let us briefly discuss the transition to chaos of the above Eulerian equations. Franceschini and coworkers have numerically studied this truncated model with $F=5$ and $F=7[9,27]$. The forcing were restricted to the $3^{\text {th }}$ mode $f_{j}=\operatorname{Re} \delta_{j, 3}[27]$. For $F=5$ and $\operatorname{Re}<\operatorname{Re}_{1}=22.85 \ldots$, there are four stable stationary solutions, say $\widehat{\mathbf{Q}}$, and $\lambda_{\mathrm{E}}<0$. At $\mathrm{Re}=\mathrm{Re}_{1}$, these solutions become unstable, via a Hopf bifurcation [29], i.e. four stable periodic orbits appear, and therefore $\lambda_{\mathrm{E}}=0$. For $\operatorname{Re}_{1}<\operatorname{Re}<\operatorname{Re}_{2}=28.41 \ldots$, one thus finds the stable limit cycles:

$$
\mathbf{Q}(t)=\widehat{\mathbf{Q}}+\left(\operatorname{Re}-\operatorname{Re}_{1}\right)^{1 / 2} \delta \mathbf{Q}(t)+O\left(\operatorname{Re}-\mathrm{Re}_{1}\right)
$$

where $\delta \mathbf{Q}(t)$ is periodic with period

$$
T(\mathrm{Re})=T_{0}+O\left(\operatorname{Re}-\mathrm{Re}_{1}\right) \quad T_{0}=0.7328 \ldots
$$

At $R e=R_{2}$, these limit cycles lose stability and there is a period doubling cascade towards Eulerian chaos.

Let us now discuss the Lagrangian behavior of a fluid particle for the above flow. For $\operatorname{Re}<\operatorname{Re}_{1}$, the stream function is asymptotically stationary, $\psi(\mathbf{x}, t) \rightarrow \widehat{\psi}(\mathbf{x})$, and the corresponding one-dimensional Hamiltonian is time-independent, therefore Lagrangian trajectories are regular. For $\mathrm{Re}=\mathrm{Re}_{1}+\epsilon$ the asymptotic stream function becomes time dependent 


$$
\psi(\mathbf{x}, t)=\widehat{\psi}(\mathbf{x})+\sqrt{\epsilon} \delta \psi(\mathbf{x}, t)+O(\epsilon)
$$

where $\widehat{\psi}(\mathbf{x})$ is given by $\widehat{\mathbf{Q}}$ and $\delta \psi$ is periodic in $\mathbf{x}$ and in $t$ with period $T$. The region of phase space, here the real two-dimensional space, adjacent to a separatrix is very sensitive to perturbations, even of very weak intensity. Figure 2 shows the structure of the separatrices (i.e. the orbits of infinite period connecting hyperbolic fixed points) at $\operatorname{Re}=\mathrm{Re}_{1}-0.05$. One can observe the presence of two kinds of separatrices: the isolated "eights" labeled by A and the connected periodic separatrices, labeled with B.

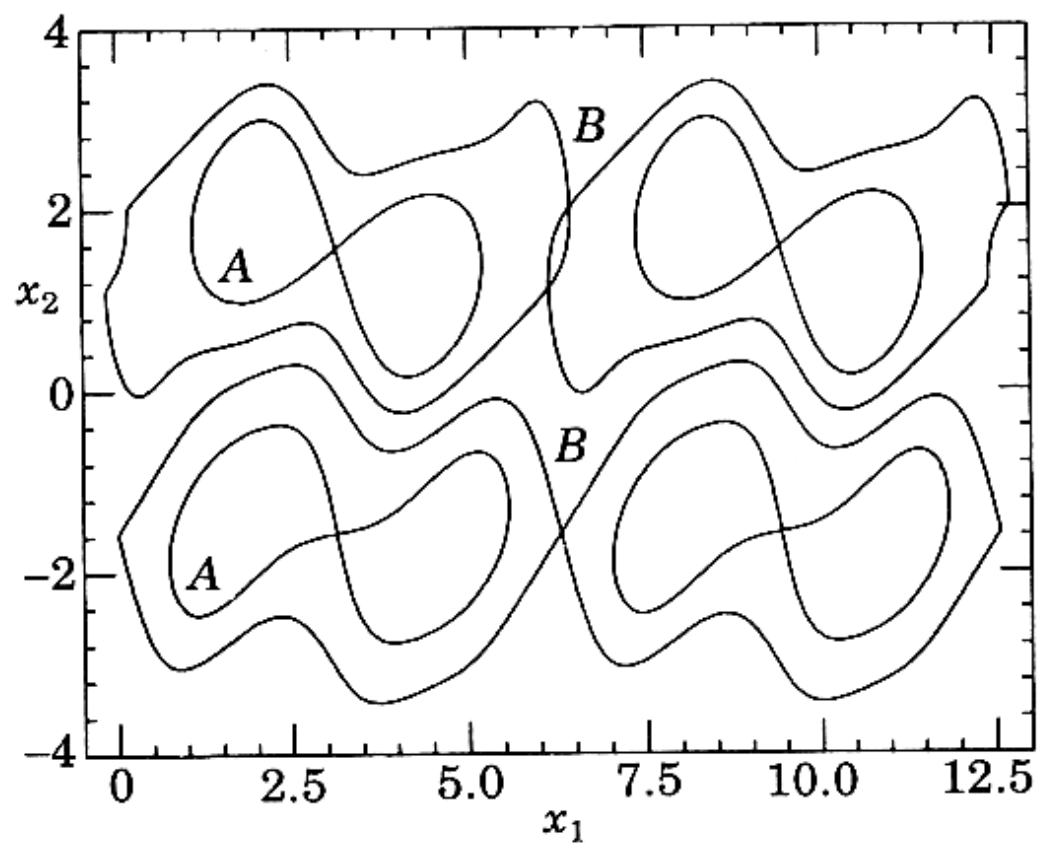

FIG. 2. Structure of the separatrices in the 5-mode model (22) with $R e=R_{1}-0.05$.

Generically, in one-dimensional Hamiltonian systems, a periodic perturbation gives origin to chaotic motion in a domain containing the hyperbolic fixed points $[14,34]$. A method due to Melnikov [34] allows to prove the existence of the chaotic layer in the phase space. However such a technique is not straightforward (one has to compute explicitly an integral which can involve complicated functions). On the other hand we have a rather strong numerical evidence for the existence of the chaotic regions, see Figure 3. 
Chaotic and regular motion for small $\epsilon=\mathrm{Re}_{1}-\mathrm{Re}$ can be studied by the Poincaré map

$$
\mathbf{x}(n T) \rightarrow \mathbf{x}(n T+T)
$$

where (at a given $\epsilon$ ) the period $T(\epsilon)$ is computed numerically. The size of the stochastic layers rapidly increase with $\epsilon$. At $\epsilon=\epsilon_{\mathrm{c}} \approx 0.7$ they overlap, according to the resonance overlap mechanism introduced by Chirikov [14], and it is practically impossible to distinguish between regular and chaotic zones. At $\epsilon>\epsilon_{\mathrm{c}}$ there is always diffusive motion.

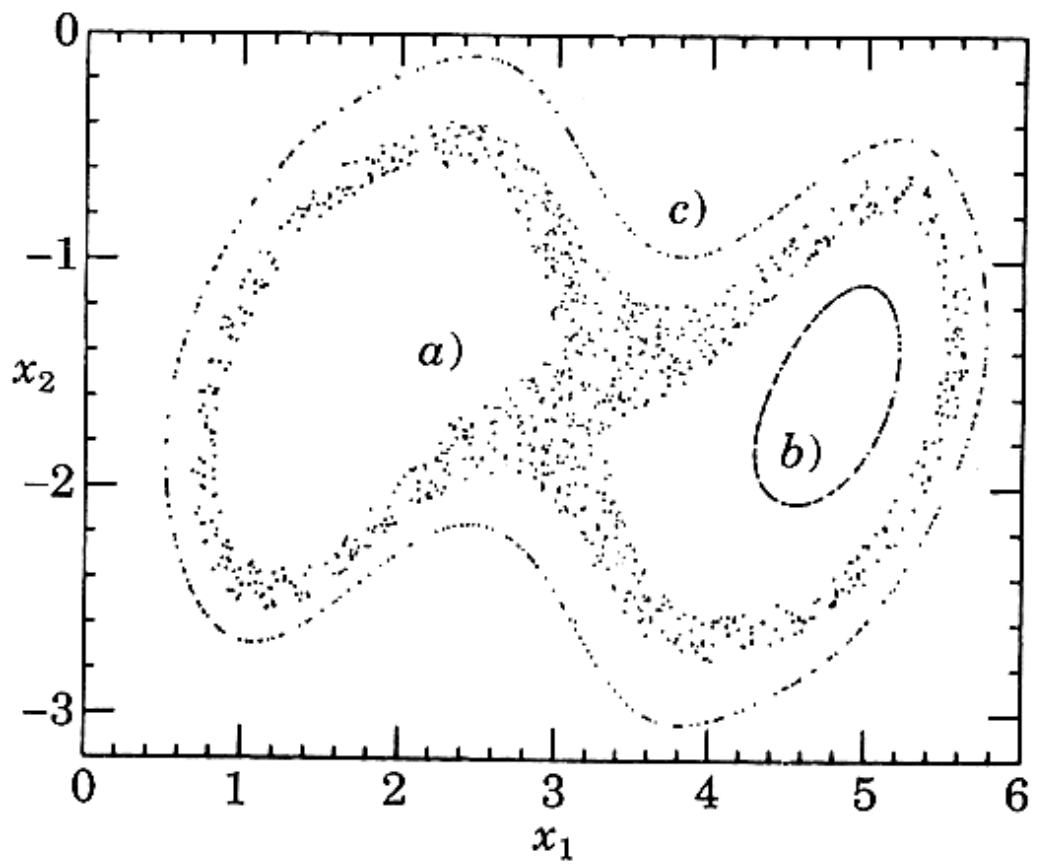

FIG. 3. Poincaré map for three trajectories of the 5-mode model with $\operatorname{Re}=\operatorname{Re}_{1}+0.05$. The initial conditions are selected close to a separatrix, case a) $\left(x_{1}(0)=3.2, x_{2}(0)=-1.6\right)$, or far from the separatrices, cases b) $\left(x_{1}(0)=4.3, x_{2}(0)=-2.0\right)$ and c) $\left(x_{1}(0)=4.267, x_{2}(0)=-3.009\right)$.

We stress that this scenario for the onset of Lagrangian chaos in two-dimensional incompressible fluids is generic and does not depend on the particular truncated model. In fact, it is only related to the appearance of stochastic layers under the effects of small timedependent perturbations in one-dimensional (i.e. with two degrees of freedom) integrable Hamiltonian systems. As consequence of general features of one-dimensional Hamiltonian systems we expect that a stationary stream function becomes time periodic through a Hopf 
bifurcation as occurs for all known truncated models of Navier-Stokes equations.

We have seen that there is no simple relation between Eulerian and Lagrangian behaviors, i.e. there is no simple relation between $\lambda_{L}$ and $\lambda_{E}$. In the following, we shall discuss two important points:

- (i) what are the effects on the Lagrangian chaos of the transition to Eulerian chaos, i.e. from $\lambda_{\mathrm{E}}=0$ to $\lambda_{\mathrm{E}}>0$.

- (ii) whether a chaotic velocity field $\left(\lambda_{\mathrm{E}}>0\right)$ always implies an erratic motion of fluid particles.

The first point can be studied again within the $F=5$ modes model (23). Increasing Re, the limit cycles bifurcate to new double period orbits followed by a period doubling transition to chaos and a strange attractor appears at $\operatorname{Re}_{c} \approx 28.73$, where $\lambda_{\mathrm{E}}$ becomes positive. These transitions have no signature on Lagrangian behavior, as shown in Figure 4, i.e. the onset of Eulerian chaos has no influence on Lagrangian properties.

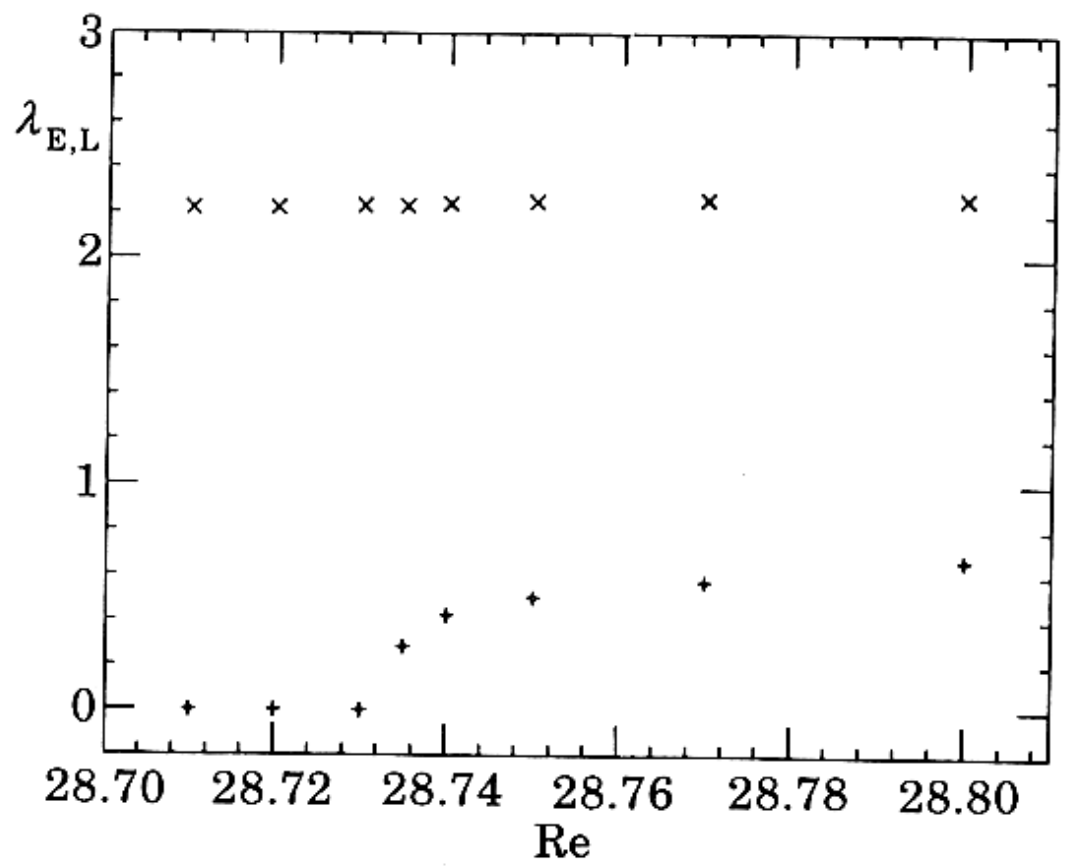

FIG. 4. Lyapunov exponents $\lambda_{E}(+)$ and $\lambda_{L}(\times)$ as function of Re around $\operatorname{Re}_{c}$, for the 5-mode model. 
This feature should be valid in most situations, since it is natural to expect that in generic cases there is a strong separation of the characteristic times for Eulerian and Lagrangian behaviors.

The second point - the conjecture that a chaotic velocity field always implies chaotic motion of particles - looks very reasonable. Indeed, it appears to hold in many systems [15]. Nevertheless, one can find a class of systems where this is false, e.g. the equations (15), (16) may exhibit Eulerian chaoticity $\lambda_{\mathrm{E}}>0$, even if $\lambda_{\mathrm{L}}=0[6]$.

\section{B. Lagrangian chaos in point-vortex systems}

Let us consider again the two-dimensional Euler equation. We assume that the vorticity $\omega=\nabla \times \mathbf{v}$, namely its component $\omega(\mathbf{r}, t)$ perpendicular to the plane of the flow, at the initial time $t=0$ is localized on $N$ point-vortices:

$$
\omega(\mathbf{r}, 0)=\sum_{i=1}^{N} \Gamma_{i} \delta\left(\mathbf{r}-\mathbf{r}_{i}(0)\right),
$$

where $\Gamma_{i}$ is the circulation of the $i$-th vortex. As consequence of the Kelvin's circulation theorem, which states that in an ideal incompressible fluid, in absence of forcings, the circulation around a closed material curve moving with the fluid is constant, one has that, at any time, $\omega(\mathbf{r}, t)$ must still remain localized on $N$ point-vortices:

$$
\omega(\mathbf{r}, t)=\sum_{i=1}^{N} \Gamma_{i} \delta\left(\mathbf{r}-\mathbf{r}_{i}(t)\right),
$$

where $\mathbf{r}_{i}=\left(x_{i}, y_{i}\right)$ evolves according to:

$$
\begin{gathered}
\Gamma_{i} \frac{d x_{i}}{d t}=\frac{\partial H}{\partial y_{i}} \\
\Gamma_{i} \frac{d y_{i}}{d t}=-\frac{\partial H}{\partial x_{i}}
\end{gathered}
$$

where

$$
H=-\frac{1}{4 \pi} \sum_{i \neq j} \Gamma_{i} \Gamma_{j} \ln r_{i j}
$$


and $r_{i j}^{2}=\left(x_{i}-x_{j}\right)^{2}+\left(y_{i}-y_{j}\right)^{2}$.

Therefore the motion of $N$ point vortices is described by a Hamiltonian system whose canonical coordinates are $\left(x_{i}, \Gamma_{i} y_{i}\right)$. Because of the presence of global conserved quantities, $\sum_{i} \Gamma_{i} x_{i}=$ const., $\sum_{i} \Gamma_{i} y_{i}=$ const., $\sum_{i} \Gamma_{i}\left(x_{i}^{2}+y_{i}^{2}\right)=$ const. and $H=$ const., a system of three vortices is integrable and there is no exponential divergence of nearby trajectories in phase space [1]. For $N \geq 4$, numerical studies show that, apart from non generic initial conditions and/or values of the parameters $\Gamma_{i}$, the system is chaotic [1].

The motion of a passively advected particle located in $(x(t), y(t))$ in the velocity field defined by (30-31) is given by

$$
\begin{gathered}
\frac{d x}{d t}=-\sum_{i} \frac{\Gamma_{i}}{2 \pi} \frac{y-y_{i}}{R_{i}^{2}} \\
\frac{d y}{d t}=\sum_{i} \frac{\Gamma_{i}}{2 \pi} \frac{x-x_{i}}{R_{i}^{2}}
\end{gathered}
$$

where $R_{i}^{2}=\left(x-x_{i}\right)^{2}+\left(y-y_{i}\right)^{2}$.

It is interesting to discuss the analogy between point-vortex systems and Celestial Mechanics. We notice that the problem of passive particles advected by $N$ vortices is formally equivalent to the case of $N+1$ vortices with $\Gamma_{N+1}=0$, in particular the case $N=3$ is integrable. In Celestial Mechanics, in the restricted 3-body problem the asteroid has a negligible mass while the sun and the planet follow Keplerian orbits. Therefore one has that the problem of a passive particle (i.e. a vortex with negligible circulation) advected by three vortices is rather similar to the restricted 3-body problem. The system (30), (31), (33) and (34), with $N \geq 4$, corresponds, in Celestial Mechanics, to the case of sun, two or more planets and an asteroid.

Let us first consider the motion of advected particles in a three-vortices (integrable) system in which $\lambda_{E}=0$. In this case, the stream function for the advected particle is periodic in time and the expectation is that the advected particles may display chaotic behavior. The typical trajectories of passive particles which have initially been placed respectively in close proximity of a vortex center or in the background field between the vortices display 
a very different behavior. The particle seeded close to the vortex center displays a regular motion around the vortex and thus $\lambda_{L}=0$; by contrast, the particle in the background field undergoes an irregular and aperiodic trajectory, and $\lambda_{L}$ is positive.

We now discuss a case where the Eulerian flow is chaotic i.e. $N=4$ point vortices. Let us consider again the trajectory of a passive particle deployed in proximity of a vortex center. As before, the particle rotates around the moving vortex. The vortex motion is chaotic; consequently, the particle position is unpredictable on long times as is the vortex position. Nevertheless, the Lagrangian Lyapunov exponent for this trajectory is zero (i.e. two initially close particles around the vortex remain close), even if the Eulerian Lyapunov exponent is positive, see Figure 5.
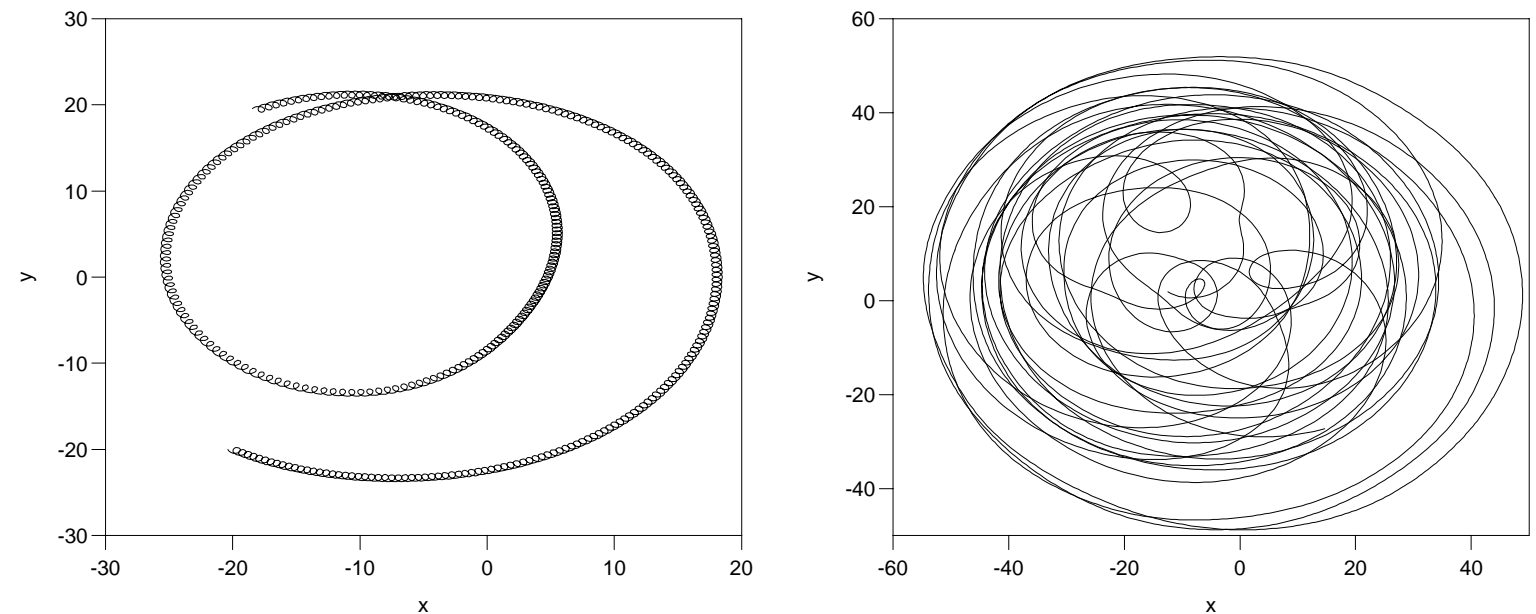

FIG. 5. Particle trajectories in the four-vortex system. Eulerian dynamics in this case is chaotic. The left panel shows a regular Lagrangian trajectory while the right panel shows a chaotic Lagrangian trajectory. The different behavior of the two particles is due to different initial conditions: particles initially close to one vortex (left panel) and particles initially distant from the vortices (right panel).

This result indicates once more that there is no strict link between Eulerian and Lagrangian chaoticity.

One may wonder whether a much more complex Eulerian flow, such as $2 D$ turbulence, may give the same scenario for particle advection: i.e. regular trajectories close to the 
vortices and chaotic behavior between the vortices. It has been shown that this is indeed the case [6] and that the chaotic nature of the trajectories of advected particles is not strictly related to the complex time evolution of the turbulent flow.

We have seen that there is no general link between Lagrangian and Eulerian chaos. In the typical situation Lagrangian chaos may appear also for regular velocity fields. However, it is also possible to have the opposite situation, with $\lambda_{\mathrm{L}}=0$ in presence of Eulerian chaos, as in the example of Lagrangian motion inside vortex structures. As an important consequence of this discussion we remark that it is not possible to separate Lagrangian and Eulerian properties in a measured trajectory, e.g. a buoy in the oceanic currents [33]. Indeed, using the standard methods for data analysis [18], from Lagrangian trajectories one extracts the total Lyapunov exponent $\lambda_{\mathrm{T}}$ and not $\lambda_{\mathrm{L}}$ or $\lambda_{\mathrm{E}}$.

\section{ASYMPTOTIC TIME BEHAVIOR: TRANSPORT AND DIFFUSION}

Let us now discuss the asymptotic behavior, i.e. at long times and large spatial scales, of the Lagrangian trajectories. The simplest model of diffusion is the Brownian motion, the erratic movement of a grains suspended in a liquid observed by the botanist Robert Brown as early as in 1827. After the fundamental work of Einstein [16], Langevin [25] and Smoluchovski [36], Brownian motion became the prototypical example of stochastic process.

From a discrete random walk model, i.e. a walker that moves randomly making jumps of $\pm \Delta x$ every time step $\Delta t$, it is easy to obtain the diffusion equation:

$$
\frac{\partial p(x, t)}{\partial t}=D \frac{\partial^{2} p(x, t)}{\partial x^{2}}
$$

where one takes the limit $\Delta x, \Delta t \rightarrow 0$ in such a way that $(\Delta x)^{2} / \Delta t \rightarrow 2 D$ (the factor 2 is purely conventional). The solution to (35) is readily obtained as

$$
p(x, t)=\frac{1}{\sqrt{4 \pi D t}} \exp \left(-\frac{x^{2}}{4 D t}\right),
$$

with $\left\langle x^{2}\right\rangle=2 D t$. Diffusion equation (35) is here written for the probability $p(x, t)$ of observing a marked particle (a tracer) in position $x$ at time $t$. The same equation can have 
another interpretation, in which $p(x, t)=\theta(x, t)$ represents the concentration of a scalar quantity (marked fluid, temperature, pollutant) as function of time. The only difference is, of course, in the normalization.

It is rather clear that time decorrelation is the key ingredient for normal diffusion. In the random walker model it is a consequence of randomness: the jumps are random uncorrelated variables and this assures the applicability of central limit theorem. But we can have a finite time correlation and thus diffusion also without randomness. A popular example is the following symplectic system (standard map [14]):

$$
\begin{aligned}
& J(t+1)=J(t)+K \sin \theta(t) \\
& \theta(t+1)=\theta(t)+J(t+1) .
\end{aligned}
$$

This map is a paradigmatic model which shows the generic behavior of chaotic symplectic systems. In particular one has large-scale chaotic behavior for $K>K_{c} \simeq 0.9716$ and, as a consequence of deterministic chaos, $J(t)$ has diffusive behavior. For long times, $J(t)$ is large and thus the angle $\theta(t)$ rotates rapidly. In this limit, we can assume that at each step $\theta(t)$ decorrelates and thus write

$$
J(t)^{2}=K^{2}\left(\sum_{t^{\prime}=1}^{t} \sin \theta\left(t^{\prime}\right)\right)^{2} \simeq K^{2}\left\langle\sin ^{2} \theta\right\rangle t=2 D t .
$$

The diffusion coefficient $D$, in the random phase approximation, i.e. assuming that $\sin \theta(t)$ is not correlated with $\sin \theta\left(t^{\prime}\right)$ for $t \neq t^{\prime}$, is obtained by the above expression as $D_{R P A}=K^{2} / 4$. As a consequence of non trivial dynamical effects (long memory due to the presence of accelerator modes) the random phase approximation is rather rough. Nevertheless, at least at qualitative level, this is able to grasp the basic ingredients of the diffusion process.

The two examples discussed above are in completely different classes: stochastic for the random walk and deterministic for the standard map (37). Despite this difference in the microscopic dynamics, both lead to a macroscopic diffusion equation and Gaussian distribution. This suggests how the diffusion equation (35) is of general applicability, and, therefore, a diffusive behavior at macroscopic scale says nothing on the underlying microscopic dynamics. 
Let us now consider the more complex situation of dispersion in a non-steady fluid with velocity field $\mathbf{v}(\mathbf{x}, t)$. For simplicity will we consider incompressible flow (i.e. $\nabla \cdot \mathbf{v}=0$ ) which can be laminar or turbulent. In presence of $\mathbf{v}(\mathbf{x}, t)$, the diffusion equation (35) becomes the advection-diffusion equation for the concentration $\theta(\mathbf{x}, t)(1)$. This equation is linear in $\theta$ but nevertheless it can display very interesting and non trivial properties even in presence of simple velocity fields, as a consequence of Lagrangian chaos.

Let us start with some general considerations. We know that in physical systems the molecular diffusivity is typically very small. Thus in (1) the advection term dominates over diffusion. This is quantified by the Peclet number, which is the ratio of the typical value of the advection term to the diffusive term

$$
\mathrm{Pe}=\frac{v_{0} l_{0}}{D}
$$

where $v_{0}$ is the typical velocity at the typical scale of the flow $l_{0}$. With $\tau_{0} \simeq l_{0} / v_{0}$ we will denote the typical correlation time of the velocity.

The central quantity in the following discussion is the concept of eddy diffusivity. The idea is rather simple and dates back to the classical work of Taylor [38]. To illustrate this concept, let us consider a Lagrangian description of dispersion in which the trajectory of a tracer $\mathbf{x}(t)$ is given by (2). Being interested in the limit $\mathrm{Pe} \rightarrow \infty$, in the following we will neglect, just for simplicity, the molecular diffusivity $D$, which is generally much lesser that the effective dynamical diffusion coefficient.

Starting from the origin, $\mathbf{x}(0)=0$, and assuming $\langle\mathbf{v}\rangle=0$ we have $\langle\mathbf{x}(t)\rangle=0$ forever. The square displacement, on the other hand, grows according to

$$
\frac{d}{d t}\left\langle\frac{1}{2} \mathbf{x}(t)^{2}\right\rangle=\left\langle\mathbf{x}(t) \cdot \mathbf{v}_{L}(t)\right\rangle=\int_{0}^{t}\left\langle\mathbf{v}_{L}(s) \cdot \mathbf{v}_{L}(t)\right\rangle d s
$$

where we have introduced, for simplicity of notation, the Lagrangian velocity $\mathbf{v}_{L}(t)=$ $\mathbf{v}(\mathbf{x}(t), t)+\sqrt{2 D_{0}} \eta(t)$, where the term $\eta(t)$ is a three-dimensional, $\delta$-correlated, normally distributed, stochastic variable, with zero mean and unit variance, and $D_{0}=l_{0} v_{0}$. Let us define the Lagrangian correlation time $\tau_{L}$ from 


$$
\int_{0}^{\infty}\left\langle\mathbf{v}_{L}(s) \cdot \mathbf{v}_{L}(0)\right\rangle d s=\left\langle\mathbf{v}_{L}(0)^{2}\right\rangle \tau_{L}
$$

and assume that the integral converge so that $\tau_{L}$ is finite. From $(40)$, for $t \gg \tau_{L}$ we get

$$
\left\langle\mathbf{x}(t)^{2}\right\rangle=2 \tau_{L}\left\langle\mathbf{v}_{L}^{2}\right\rangle t
$$

i.e. diffusive behavior with diffusion coefficient (eddy diffusivity) $D^{E}=\tau_{L}\left\langle\mathbf{v}_{L}^{2}\right\rangle$.

This simple derivation shows, once more, that diffusion has to be expected in general in presence of a finite correlation time $\tau_{L}$. Coming back to the advection-diffusion equation (1), the above argument means that, for $t \gg \tau_{L}$, we expect that the evolution of the concentration, for scales larger than $l_{0}$, can be described by an effective diffusion equation, i.e.

$$
\frac{\partial\langle\theta\rangle}{\partial t}=D_{i j}^{E} \frac{\partial^{2}\langle\theta\rangle}{\partial x_{i} \partial x_{j}} .
$$

The coefficients $D_{i j}^{E}$ are related to the statistical properties of the trajectories:

$$
D_{i j}^{E}=\lim _{t \rightarrow \infty} \frac{1}{2 t}\left\langle x_{i}(t) x_{j}(t)\right\rangle
$$

The original idea of a hydrodynamic analysis to derive eq. (43) from eq. (1) is due to Maxwell. However, only in recent years the multiscale techniques allowed for a rigorous derivation of the Fick's equation (43), as well as its range of validity, and a systematic method for the computation of the effective diffusion coefficients $D_{i j}^{E}[8,28]$. The idea behind this method has close connection with Lindstedt-Poincaré's perturbation theory, in Celestial Mechanics, where the asymptotic effects of the nonlinearities, in an ordinary differential equation, are treated with the introduction of (slow) frequencies which depend on the initial conditions and the nonlinear terms [32]. Since in the diffusion process one is interested in large scales and long times, let us assume the existence of some (small) parameter $\epsilon$. One can thus introduce, in addition to the natural (fast) variables $\mathbf{x}$ and $t$, slow variables $\mathbf{X}=\epsilon \mathbf{x}$ and $T=\epsilon^{2} t$. Treating the two sets of variables (slow and fast) as independent, and expanding $\theta$ in a perturbative series 


$$
\theta(\mathbf{x}, t ; \mathbf{X}, T)=\theta^{(0)}(\mathbf{X}, T)+\epsilon \theta^{(1)}(\mathbf{x}, t ; \mathbf{X}, T)+\ldots
$$

one has a set of equations for $\theta^{(0)}, \theta^{(1)}, \ldots$. Using the linear structure of the problem and imposing the solvability conditions $[8,28]$ (this is the analogue of the suppression of the secular terms in the multiple-scale approach for the ordinary differential equations [7]) one has that $\theta^{(0)}$ obeys to the Fick's equation (43).

The computation of the eddy diffusivity for a given Eulerian flow is not an easy task. However it is possible to show that

$$
D_{i j}^{E}=D \delta_{i j}-\frac{1}{2}\left[\left\langle v_{i} w_{j}\right\rangle+\left\langle v_{j} w_{i}\right\rangle\right]
$$

where the vector $\mathbf{w}$ satisfies the equation

$$
\partial_{t} \mathbf{w}+(\mathbf{v} \cdot \nabla) \mathbf{w}=D \nabla^{2} \mathbf{w}-\mathbf{v}
$$

Let us stress that with the multiple-scale approach one has that the asymptotic behavior of the transport equation (1) is well approximated by the Fick's law (43) where the eddy diffusivity tensor $D^{E}$ depends on the advection field $\mathbf{v}$. This is rather close, both from a technical and conceptual point of view, to the Lindstedt-Poincaré method in Mechanics where the asymptotic effect of the nonlinear terms is to induce a renormalization of the frequencies, which depend on the initial conditions and the nonlinear terms, see [35] vol. II, chap. 9, and [32]. The explicit computation can be done only in the case of simple flows $[28,8]$. On the other hand, in the general case, it is relatively simple [8] to give some bounds, the simplest one being $\operatorname{tr}\left(D^{E}\right) \geq D \cdot d$, with $d$ equal to the space dimension, i.e. the presence of an incompressible velocity field enhances large-scale transport.

Diffusion equation (43) is the typical long-time behavior in generic flow. However there exists, also, the possibility of the so-called anomalous diffusion, i.e. when the spreading of particle do not grow linearly in time, but with a power law:

$$
\left\langle x^{2}(t)\right\rangle \sim t^{2 \nu}
$$

with $\nu \neq 1 / 2$. The case $\nu>1 / 2$ (formally $D^{E}=\infty$ ) is called superdiffusion; subdiffusion, i.e. $\nu<1 / 2$ (formally $D^{E}=0$ ), is possible only for compressible velocity fields. 
Super-diffusion arises when the Taylor argument for deriving (42) fails and formally $D^{E} \rightarrow \infty$. This can be due to one of the following mechanisms:

a) the divergence of $\left\langle\mathbf{v}_{L}^{2}\right\rangle$ (which is the case of Lévy flights), or

b) the lack of decorrelation, i.e. $\left\langle\mathbf{v}_{L}(t) \mathbf{v}_{L}(0)\right\rangle \sim t^{-\beta}$, with $\beta \leq 1$, and thus $\tau_{L} \rightarrow \infty$ (Lévy walks).

The second case is more physical and it is related to the existence of strong correlations in the dynamics, even at long times and large scales.

Avellaneda and Majda [28] obtained a very general important result for the validity of eq. (43) in an incompressible velocity field. If the molecular diffusion coefficient $D$ is non zero and the infrared contribution to $\mathbf{v}$ is weak enough, namely

$$
\int d \mathbf{k} \frac{\left\langle|\widehat{\mathbf{v}}(\mathbf{k})|^{2}\right\rangle}{k^{2}}<\infty
$$

where $\langle\cdot\rangle$ indicates the time average and $\widehat{\mathbf{v}}$ is the Fourier transform of $\mathbf{v}$, then one has standard diffusion, i.e. the $D_{i j}^{E}$ are finite. Therefore there exist two possible origins for superdiffusion:

I) $D>0$ and, in order to violate (49), a velocity field with very long spatial correlations;

II) $D=0$ and strong correlation between $\mathbf{v}[\mathbf{x}(t)]$ and $\mathbf{v}[\mathbf{x}(t+\tau)]$ at large $\tau$.

An example of anomalous diffusion, according to the mechanism I, is a $2 D$ random shear, i.e. $\mathbf{v}=(v(y), 0)$ where $v(y)$ is a random function obtained with a spatial Brownian process. For such a case it is possible to show that $\left\langle x^{2}(t)\right\rangle \sim t^{3 / 2}$ and, in addition, the pdf is non Gaussian $[28,22,11]$ :

$$
p(x, t) \sim t^{-3 / 2} \exp \left(-\frac{c\left|x^{4 / 3}\right|}{t^{2}}\right) .
$$

Let us now briefly discuss anomalous diffusion due to the mechanism II: consider a simple model simulating Rayleigh-Bénard convection:

$$
\Psi(x, y, t)=\Psi_{0} \sin (x+B \sin (\omega t)) \sin (y)
$$

The even oscillatory instability is accounted for the term $B \sin (\omega t)$ representing the lateral oscillations of the rolls. In spite of its apparent simplicity, this model is able to capture the 
essential features of the convective problem [37]. For a generic value of $\omega$ one has standard diffusion, and the only role of the advection term is to enhance $D^{E}$. For special values of $\omega$ (resonance with the characteristic frequency of the passive particle motion in the steady case) one has, in the limit $D \rightarrow 0$, superdiffusion, i.e. $\left\langle x^{2}(t)\right\rangle \sim t^{2 \nu}$ with $\nu>1 / 2$.

A similar behavior, i.e. standard diffusion for generic values of the control parameters and superdiffusion for specific values, has been observed for the standard map. In this system at certain values of $K$ the accelerator modes induce a superdiffusion with a non gaussian shape of the pdf $[21,11]$.

Therefore, on the basis of the above results (and similar results found for other symplectic systems [26]), one can say that the mechanism II is non generic. Introducing a small perturbation $O(\epsilon)$ in the evolution law, superdiffusion disappears i.e. it is present only for a transient time up to $t_{*} \sim \epsilon^{-\alpha}$. At longer times standard diffusion takes place with a rather large diffusion coefficient: we can say that, even if the true (i.e. asymptotic) anomalous diffusion is very rare, its ghost is still visible [11].

\section{CONCLUSIONS}

The recent advances in the understanding of the chaotic behavior and the (asymptotic) diffusive properties of Lagrangian trajectories, advected by laminar velocity fields, stem from

ideas and techniques borrowed from Poincaré's contributions to Celestial Mechanics (mainly the homoclinic intersection mechanism and multiple-scale expansion). This bears the indelible mark of the genius of Henri J. Poincaré and shows how many areas of Mathematics and Physics have been affected by his works.

We acknowledge the fruitful and friendly collaboration of many colleagues who shared with us their knowledge and research, in particular P. Castiglione, A. Celani, M. Cencini, M. Falcioni, A. Mazzino, P. Muratore-Ginanneschi, S. Musacchio and M. Vergassola. 


\section{References}

[1] H. Aref, Integrable, and turbulent vortex motion in two-dimensional flows, Ann. Rev. Fluid Mech. 15, 345 (1983).

[2] H. Aref, Stirring by chaotic advection, J. Fluid Mech. 143, 1 (1984).

[3] H. Aref and S. Balachandar, Chaotic advection in a Stokes flow, Phys. Fluids, 29, 3515 (1986).

[4] V.I. Arnold, Sur la topologie des ecoulements stationnaires des fluides parfaits, C. R. Acad. Sci. Paris A, 261, 17 (1965).

[5] V.I. Arnold and B.A. Khesin, Topological methods in hydrodynamics, Applied Math. Sciences, 125, Springer-Verlag, Berlin (1998).

[6] A. Babiano, G. Boffetta, A. Provenzale and A. Vulpiani, Chaotic advection in point vortex models and two-dimensional turbulence, Phys. Fluids A 6, 2465 (1994).

[7] C. Bender and S. A. Orszag, Advanced Mathematical Methods for Scientists and Engineers, McGraw-Hill, New York (1978).

[8] L. Biferale, A. Crisanti, M. Vergassola and A. Vulpiani, Eddy diffusivities in scalar transport, Phys. Fluids 7, 2725 (1995).

[9] C. Boldrighini and V. Franceschini, A five-mode truncation of the plane Navier-Stokes equations, Commun. Math. Phys., 64, 159 (1979).

[10] J.P. Bouchaud and A. Georges, Anomalous diffusion in disordered media: statistical mechanisms, models and physical applications, Phys. Rep. 195, 127 (1990).

[11] P. Castiglione, A. Mazzino, P. Muratore-Ginanneschi and A. Vulpiani, On the strong anomalous diffusion, Physica D, 134, 75 (1999).

[12] J. Chaiken, C.K. Chu, M. Tabor and Q.M. Tan, Lagrangian turbulence in Stokes flow, Phys. 
Fluids, 30, 687 (1987).

[13] S. Chandrasekhar, Stochastic problems in Physics and Astronomy, Rev. Mod. Phys. 15, 1 (1943).

[14] B.V. Chirikov, A universal instability of many dimensional oscillator systems, Phys. Rep. 52, $263(1979)$.

[15] A. Crisanti, M. Falcioni, G. Paladin and A. Vulpiani, Lagrangian chaos: transport, mixing and diffusion in fluids, Riv. Nuovo Cim. 14, 1 (1991).

[16] A. Einstein, On the movement of small particles suspended in a stationary liquid demanded by the molecular kinetic theory of heat, Ann. Phys. (Leipzig) 17, 549 (1905).

[17] M. Falcioni, G. Paladin and A. Vulpiani, Regular and chaotic motion of fluid-particles in two-dimensional fluids, J. Phys. A: Math. Gen., 21, 3451 (1988).

[18] P. Grassberger and I. Procaccia, Estimation of the Kolmogorov entropy from a chaotic signal, Phys. Rev. A, 28, 2591 (1983).

[19] M. Hénon, Sur la topologie des lignes de courant dans un cas particulier, C. R. Acad. Sci. Paris A, 262, 312 (1966).

[20] P.J. Holmes, J.L. Lumley and G. Berkooz, Turbulence, coherent structures, dynamical systems and symmetry, Cambridge University Press, Cambridge (1996).

[21] Y.H. Ichikawa, T. Kamimura and T. Hatori, Stochastic diffusion in the standard map, Physica D, 29247 (1987).

[22] M.B. Isichenko, Percolation, statistical topography, and transport in random media, Rev. Mod. Phys. 64961 (1992).

[23] H. Lamb, Hydrodynamics, New York Dover Publ., New York (1945).

[24] L.D. Landau and L. Lifshitz, Fluid Mechanics, New York Pergamon Press, New York (1987). 
[25] P. Langevin, Sur la Theorie du Mouvement Brownien, Comptes Rendus 146, 530 (1908).

[26] P. Leboeuf, Normal and anomalous diffusion in a deterministic area-preserving map, Physica D, 116, 8 (1998).

[27] J. Lee, Triad-angle locking in low-order models of the 2D Navier-Stokes equations, Physica D, 24, 54 (1987).

[28] A.J. Majda and P.R. Kramer, Simplified models for turbulent diffusion: theory, numerical modeling and physical phenomena, Phys. Rep. 314, 237 (1999).

[29] J.E. Marsden and M. McCracken, The Hopf bifurcation and its applications, MIT Press, Cambridge, Mass. (1975).

[30] M.R. Maxey and J.J. Riley, Equation of motion for a small rigid sphere in a non uniform flow, Phys. Fluids 26, 883 (1983).

[31] H.K. Moffat, Transport effects associated with turbulence, with particular attention to the influence of helicity, Rep. Prog. Phys. 46, 621 (1983).

[32] J.A. Murdock, Perturbations: theory and methods, J. Wiley, New York (1991).

[33] A.R. Osborne, A.D. Kirwan, A. Provenzale and L. Bergamasco, A Search for Chaotic Behaviour in Large and Mesoscale Motions in the Pacific Ocean, Physica D 23, 75 (1986).

[34] E. Ott, Chaos in dynamical systems, Cambridge University Press, Cambridge (1993).

[35] H. J. Poincaré, Les Méthodes Nouvelles de la Mécanique Céleste, Gauthier-Villars, Paris (1892, 1893, 1899).

[36] M. Smoluchowski, (Euvres, Acad. Pol. Sci. Lettres (Cracovie), Librairie Polytechnique Ch. Béranger, Paris (1924).

[37] T.H. Solomon and J.P. Gollub, Passive Transport in Steady Rayleigh-Bnard Convection, Phys. Fluids 31, 1372 (1988). 
[38] G.I. Taylor, diffusion by continuous movements, Proc. London Math. Soc. 2, 196 (1921).

[39] D.J. Tritton, Physical fluid dynamics, Oxford Science Publ., Oxford (1988). 